\title{
A Review of Various Techniques of Central Venous Catheter Insertion
}

\author{
Shallu Chaudhary ${ }^{1}$, Major Amit Atwal ${ }^{2}$ \\ ${ }^{1}$ Medical Officer, Health \& Family Welfare Department, Himachal Pradesh, India \\ ${ }^{2}$ Medical Officer, Military Hospital Jutogh Cantt, Shimla, Himachal Pradesh, India \\ Corresponding Author: Major Amit Atwal
}

\begin{abstract}
Central venous catheterization is a standard procedure used in the resuscitation of critically ill patients. There are different routes of CVP insertion which are:- subclavian vein, internal jugular vein, axillary vein and femoral vein. Each route has its own set of complications like artery puncture, pneumothorax, nerve injury, infections. Initially catheters were inserted by the landmark technique using guidewire through the needle commonly called Seldinger technique. The landmark technique is found to be associated with a higher range of mechanical complications. The use of USG however has proved to improve the success rate and decrease the complications.
\end{abstract}

Keywords: Central venous catheterization, resuscitation, internal jugular vein

\section{INTRODUCTION}

Central venous catheterization is a very important part of management of critically ill patients. They are inserted by anesthesiologists, intensivists, radiologists, attending physicians and surgeons. It is the standard clinical method for monitoring central venous pressure, therapeutic interventions like securing vascular access for administration of vasoactive drugs or to initiate rapid fluid resuscitation.

Various routes of central line insertion have been defined that is:subclavian vein route, internal jugular route, axillary vein, femoral vein. However each route is associated with its own set of challenges and the overall incidence of mechanical complications ranges from 5$19 \%^{1}$. The choice of the access site depends on various factors including physician preference, ease of access, coagulation status, probable duration of catheter stay, infection rates and complication rates. Complications of central venous catheterization include arterial puncture, hematoma, pneumothorax, arterial venous fistula, air embolism, nerve injury, infections and thrombosis. The usefulness of ultrasound has demonstrated catheterization with low incidence of complications. Many studies have come up using ultrasonography guidance for central venous access showing higher success rates. Although there are studies and reports of use of USG guidance in all routes of central line access like subclavian, axillary and femoral routes, the highest evidence of usefulness has been demonstrated in internal jugular vein route.

\section{REVIEW OF LITERATURE}

Millions of central vein catheterizations are done annually by various clinicians for monitoring as well as therapeutic purposes. Central lines insertion was first described by Aubaniac ${ }^{2}$ (1952) when he inserted a needle in subclavian vein under the clavicle. Various other routes of central venous access were described subsequently in the next decade including the infraclavicular axillary vein approach, internal jugular venous route and supraclavicular approach to subclavian vein. 
Later Seldinger ${ }^{3}$ (1953) modified the technique of catheter insertion by passing a guide wire through the needle and threading the catheter over the guide wire. This technique became popular worldwide and now goes by the name 'Seldinger Technique'. Yoffa ${ }^{4}$ (1965) described supraclavicular subclavian vein catheterization where he described various landmarks and the technique ${ }^{5}$ of cannulation.

Hermosura et $\mathrm{al}^{6}$ (1966) first described the technique of percutaneous internal jugular vein cannulation. Nickalls ${ }^{7}$ (1985) described the infraclavicular approach to axillary vein cannulation. He described two surface landmarks for axillary vein cannulation, first one being three finger breadth below coracoid process and the second point at the junction of medial one third and lateral two third of clavicle.

Central venous catheterization is associated with many complications of which some can be serious and some life threatening. The incidence and associations of these complications with different approaches for central venous catheterization have been reported in literature. Tsotsolos et $\mathrm{al}^{8}$ (2015) shared the opinion on experience of the clinician in performing the procedure as a major risk factor for mechanical complications.

However, Schummer et $\mathrm{al}^{9}$ (2007) has studied central venous cannulation by landmark technique through various routes and reported a high incidence of mechanical complications even with experienced operators. He reported a significantly higher incidence of arterial puncture with IJV cannulation when compared with subclavian vein cannulation.

Anatomic considerations while inserting central line through various routes have been described in detail by Bannon et $\mathrm{al}^{10}$ (2011). In his article he described the anatomy of the central veins commonly used for catheterization, proper preparation, insertion techniques, various complications and use of ultrasound guidance.
Merrer et $\mathrm{al}^{11}$ (2001) in his study comparing the complications of femoral venous access and subclavian approach in critically ill patients reported infection rates of $19.8 \%$ in femoral venous catheterization compared to $4.5 \%$ with subclavian route. The incidence of thrombosis was also higher with femoral route. The high incidence of thrombotic complications along with the high rates of infection has made femoral route the least preferred route for central venous access.

Eisen et $\mathrm{al}^{1}$ (2006) in a retrospective study of mechanical complications in central venous catheters, observed a pneumothorax incidence of $2.3 \%$ with subclavian route whereas there was none in the jugular vein group. They concluded that the incidence of complications is higher with subclavian route and this is to be considered while choosing the approach.

There is a huge body of literature demonstrating the usefulness of ultrasound in vascular access. Ultrasound guidance was first used for vascular access by Legler et $\mathrm{al}^{12}$ (1984). He compared the use of Doppler pre-location to classic landmark technique to guide the venous cannulation. He demonstrated the need of lesser needle passes and higher success rates with Doppler pre-location. Denys et $\mathrm{al}^{13}(1993)$ in one of the earlier studies comparing ultrasound guidance and external landmark technique for IJV cannulation reported success rate of $100 \%$ using ultrasound and $88.1 \%$ using the landmark-guided technique.

Fathi et $\mathrm{al}^{14}$ (2016) in a more recent study compared anatomical landmark and USG guided internal jugular vein catheterization in patients undergoing cardiac surgery. He reported high success rates of $99.4 \%$ with USG guidance. Slama et $\mathrm{al}^{15}$ (1997) in a prospective randomized study, compared use of anatomical landmarks with use of ultrasound guidance for IJV cannulation by junior house staff. They found that IJV cannulation was successful in $100 \%$ patients in the ultrasound group and $76 \%$ in the control 
group. Ultrasound guidance has shown to decrease the venous access time, number of needle passes and the incidence of mechanical complications, improves overall and first attempt success rates. The evidence of the efficacy and usefulness of ultrasound in internal jugular vein cannulation is strong and this has prompted the adoption of ultrasound guidance in $\mathrm{NICE}^{16}$ guidelines for safe practices in 2002 .

Vogel et $\mathrm{al}^{17}$ (2015) compared long axis and short axis views for internal jugular and subclavian vein cannulations in mannequins and found that posterior wall punctures were lesser with long axis views. Moore et $\mathrm{al}^{18}$ (2014) in his article described the importance of ultrasound in the present era, not just for central venous access but also for central or peripheral arterial access and peripheral venous access. He concluded that USG guidance can increase success and decrease complications in a wide variety of vascular access procedures.

Blaivas $M$ et $\mathrm{al}^{19}$ (2003) did a randomized, observational study to compare short axis versus long axis approaches for teaching ultrasound guided vascular access on a new inanimate model. They concluded that novice USG users obtained vascular access faster using short axis approach than long axis approach on inanimate model.

Tammam TF et $\mathrm{al}^{20}$ (2013) compared two different ultrasound guided techniques (short axis versus long axis approach) and landmark technique for internal jugular vein cannulation in 90 critical care and hemodialysis patients. They concluded that short axis and long axis approaches were comparable for IJV cannulation. Furthermore, both USG guided techniques were superior to the landmark technique for insertion of CVP.

Chaudhary MS et $\mathrm{al}^{21}$ (2016) compared Ultrasound guided internal jugular vein cannulation with short and long axis approach. They postulated that higher first attempt successful cannulation and overall less complications were seen in long axis approach but the difference was not statistically significant.
Shrestha GS et $\mathrm{al}^{22}$ (2016) did a comparison between short and long axis techniques for ultrasound guided cannulation of internal jugular vein and concluded that both techniques have similar outcomes when used for IJV cannulation.

\section{CONCLUSION}

There is a huge body of literature demonstrating the various techniques of central venous catheterization over the years. The use of ultrasound has converted a blind procedure into a procedure under vision reducing the complications markedly. Thus, USG guidance should be considered in all routes of central venous access. Training and experience with the use of USG improves the success rate and decreases complications.

\section{Acknowledgement: None}

\section{Conflict of Interest: None}

\section{Source of Funding: None}

\section{Ethical Approval: Not required}

\section{REFERENCES}

1. Eisen LA, Narasimhan M, Berger JS, Mayo $\mathrm{PH}$, Rosen MJ, Schneider RF. Mechanical complications of central venous catheters. J Intensive Care Med.2006;21(1):40-46.

2. Aubaniac R. (Subclavian intravenous injection; advantages and technic). Presse Medicale. 1952;60(68):1456.

3. Seldinger SI. Catheter replacement of the needle in percutaneous arteriography; a new technique. Acta Radiol. 1953;39(5):368376.

4. Yoffa D. Suraclavicular subclavian venepuncture and catheterization. Lancet Lond Engl. 1965;(7413):614-617.

5. MacDonnell JE, Perez H, Pitts SR, Zaki SA. Supraclavicular subclavian vein catheterization: modified landmarks for needle insertion. Ann Emerg Med. 1992; 21(4):421-424.

6. Hermosura B, Vanags L, Dickey MW. Measurement of pressure during intravenous therapy. JAMA.1966;195:181. 
7. Nickalls RW. A new percutaneous infraclavicular approach to the axillary vein. Anaesthesia. 1987;42(2):151-154.

8. Tsotsolis N, Tsirgogianni K, Kioumis I et al. Pneumothorax as a complication of central venous catheter insertion. Ann Transl Med. 2015;3(3):40.

9. Schummer W, Schummer C, Rose N, Niesen WD, Sakka SG. Mechanical complications and malpositions of central venous cannulations by experienced operators. A prospective study of 1794 catheterizations in critically ill patients. Intensive Care Med. 2007;33(6):1055-1059.

10. Bannon MP, Heller SF, Rivera M. Anatomic considerations for central venous cannulation. Risk Manag Healthc Policy. 2011;4:27-39.

11. Merrer J, De Jonghe B, Golliot F, et al. Complications of femoral and subclavian venous catheterization in critically ill patients: a randomized controlled trial. JAMA.2001;286(6):700-707.

12. Legler D, Nugent M. Doppler localization of the internal jugular vein facilities central venous cannulation. Anesthesiology. 1984; 60(5): 481-482.

13. Denys BG, Uretsky BF, Reddy PS. Ultrasound-assisted cannulation of the internal jugular vein. A prospective comparison to the external landmark guided technique. Circulation. 1993;87(5):15571562.

14. Fathi M, Izanloo A, Jahanbakhsh S, et al. Central Venous Cannulation of the Internal Jugular Vein Using Ultrasound - Guided and Anatomical Landmark Techniques. Anesthesiol Pain Med. 2016;6(3).

15. Slama M, Novara A, Safavian A, Ossart M, Safar M, Fagon JY. Improvement of internal jugular vein cannulation using an ultrasound-guided technique. Intensive Care Med. 1997;23(8):916-919.
16. Guidance on the use of ultrasound locating devices for placing central venous catheters | Guidance and guidelines | NICE.

17. Vogel JA, Haukoos JS, Erickson CL, et al. Is long axis view superior to short axis view in ultrasound-guided central venous catheterization? Crit Care Med. 2015;43(4): 832-839.

18. Moore CL. Ultrasound first, second and last for vascular access. J Ultrasound Med Off J Am Inst Ultrasound Med. 2014;33(7):11351142.

19. Blaivas M, Brannam L, Fernandez E, Short axis versus Long axis approaches for teaching ultrasound guided vascular access on a new inanimate model. Acad Emerg Med. 2003 Dec;10(12):1307-11. doi: 10.1111/j.1553-2712.2003.tb00002.x.

20. Tammam TF, El-Shafey EM, Tammam HF. Ultrasound guided internal jugular vein access: Comparison between Short Axis and Long Axis Techniques. Saudi J Kidney Dis Transpl 2013;24(4):707-713.

21. Chaudhari MS, Bharat Shah S, Vithal Kamat H. Ultrasound guided internal jugular vein cannulation with short and long axis approach- Technical ease and complications. Indian Journal of Clinical Anaesthesia, 2016;3(4):546-550.

22. Shrestha GS, Gurung A, Koirala S. Comparison between long and short axis technique for ultrasound-guided cannulation of internal jugular vein. Ann Card Anaesth 2016;19:288-92.

How to cite this article: Chaudhary S, Atwal MA. A review of various techniques of central venous catheter insertion. International Journal of Science \& Healthcare Research. 2021; 6(3): 204-207. DOI: https://doi.org/10.52403/ijshr. 20210735 